\title{
Flow-orthogonal bead oscillation in a microfluidic chip with a magnetic anisotropic flux-guide array
}

\author{
Stijn van Pelt • Roy Derks • Marco Matteucci · \\ Mikkel Fougt Hansen • Andreas Dietzel
}

Published online: 17 December 2010

(C) The Author(s) 2010. This article is published with open access at Springerlink.com

\begin{abstract}
A new concept for the manipulation of superparamagnetic beads inside a microfluidic chip is presented in this paper. The concept allows for bead actuation orthogonal to the flow direction inside a microchannel. Basic manipulation functionalities were studied by means of finite element simulations and results were oval-shaped steady state oscillations with bead velocities up to $500 \mu \mathrm{m} / \mathrm{s}$. The width of the trajectory could be controlled by prescribing external field rotation. Successful verification experiments were performed on a prototype chip fabricated with excimer laser ablation in polycarbonate and electroforming of nickel flux-guides. Bead velocities up to $450 \mu \mathrm{m} / \mathrm{s}$ were measured in a $75 \mu \mathrm{m}$ wide channel. By prescribing the currents in the external quadrupole magnet, the shape of the bead trajectory could be controlled.
\end{abstract}

Electronic supplementary material The online version of this article (doi:10.1007/s10544-010-9503-5) contains supplementary material, which is available to authorized users.

S. van Pelt $(\bowtie) \cdot$ R. Derks · M. Matteucci - A. Dietzel

Department of Mechanical Engineering,

Micro- and NanoScale Engineering,

Eindhoven University of Technology,

PO Box 513, 5600 MB Eindhoven,

The Netherlands

e-mail: s.v.pelt@tue.nl

\section{F. Hansen}

Department of Micro- and Nanotechnology,

Technical University of Denmark,

DTU Nanotech, Building 345 East,

2800 Kongens Lyngby, Denmark
Keywords Superparamagnetic beads • Microfluidics • Quadrupole magnet • Shape-anisotropy • Magnetic flux-guides

\section{Introduction}

In the biomedical field, a trend has emerged from laboratory based machinery towards handheld pointof-care devices to be used by a practitioner. These devices greatly reduce time, sample volumes and costs because they can combine many complicated tasks like mixing, separation, transportation and detection in a small package (Manz et al. 1990). Current methods for biological separation are often based on superparamagnetic beads for their bio-orthogonal nature and the range of possibilities to manipulate them (Jaephil and Chong 2008; Derks et al. 2007; Aytur et al. 2006; Gundersen et al. 1992; Gehring et al. 2004). In these methods, beads are coated with a specific antibody in order to capture a targeted species. These functionalized beads are immobilized on a channel wall by applying a magnetic field. When the sample fluid is injected in the channel, the target species will diffuse and attach to the beads thereby separating the target from the fluid (Ramadan et al. 2006), see Fig. 1(a). However, at very low concentrations of $\mathrm{pmol} / \mathrm{l}$ or lower, the distance a target species has to travel becomes very large, in the $\mu \mathrm{m}$ to $\mathrm{mm}$ range (Manz et al. 1990). Therefore only low flow-rates can be used. Also, target species in the center of the channel have a lower capture probability than species close to the wall due to the laminar flow-profile that has no flow-orthogonal velocity component. The species can only travel orthogonally to the flow by means of diffusion. Furthermore, the flow-parallel 


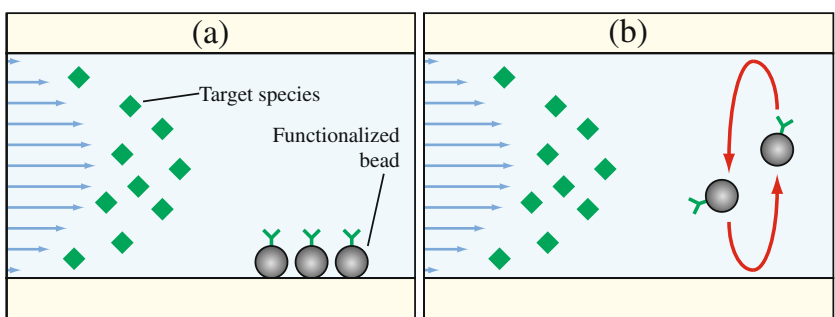

Fig. 1 The target species in the sample fluid have to attach to a bead to be separated from the fluid. In case (a) the beads are immobilized on the channel wall and target species that flow by have to diffuse towards the wall to reach a bead. When the bead is actively moved across the channel (b), the target species do not have to diffuse since the beads cover the whole channel cross section

velocity is the highest in the center of the channel which reduces the maximum flow-rate even more.

Since point-of-care devices need to give a result within minutes, a new concept is proposed in which beads are made to scan the fluid. Scanning of the fluid can be obtained by forcing the beads in an oscillating motion orthogonal to the flow, see Fig. 1(b). This way, long diffusion lengths no longer play a role and also the radial position of a target species within the channel will not influence the chance of it reaching a bead.

\section{Theory and design}

According to Derks et al. (2007), in magnetic bead motion within a fluid, the magnetic and drag forces dominate the bead motion, since at the micrometer scale, effects of gravity and inertia become very small. Therefore a bead will almost instantaneously accelerate to its terminal velocity at which the magnetic and drag force are exactly at balance with each other. It will be assumed that the beads do not saturate. This is true for beads with an effective susceptibility of $\chi_{\mathrm{b}, \text { eff }}=1.52$ and saturation magnetization of $M_{\mathrm{s}}=336 \mathrm{kA} / \mathrm{m}$ in a field with flux density $B=0.1 \mathrm{~T}$ (Fonnum et al. 2005). The magnetic force on a single bead $\mathbf{F}_{\text {mag }}$ can then be described by (Derks et al. 2007):

$\mathbf{F}_{\mathrm{mag}}=\frac{V_{\mathrm{b}} \cdot \chi_{\mathrm{b}, \mathrm{eff}}}{2 \cdot \mu_{0}} \nabla B^{2}$

Here $V_{\mathrm{b}}$ and $\chi_{\mathrm{b} \text {,eff }}$ respectively are the volume and effective magnetic susceptibility of the bead, $\mu_{0}$ is the permeability of vacuum and $B$ is the magnetic flux density in the absence of the bead. When single bead motion in a laminar flow is assumed, the drag force on a bead $\mathbf{F}_{\text {drag }}$ is given by (Derks et al. 2007):

$\mathbf{F}_{\mathrm{drag}}=-6 \cdot \pi \cdot \eta_{\mathrm{f}} \cdot r_{\mathrm{b}} \cdot \mathbf{v}_{\mathrm{b}}$
Here $\eta_{\mathrm{f}}$ is the dynamic viscosity of the carrier fluid and $r_{\mathrm{b}}$ and $\mathbf{v}_{\mathrm{b}}$ are the bead radius and velocity relative to the fluid. As follows from Eq. 1 the magnetic force on a bead is proportional to the squared field gradient $\nabla B^{2}$ and points in the same direction. In a two-pole system this means the superparamagnetic beads will move towards both the north and south poles. Bead oscillation can then be obtained by switching the magnetic field on and off. When it is turned on the beads move towards both poles, when it is turned off the fluid flow will drag the beads away from the magnetic poles (Lund-Olesen et al. 2008). This, however, results in oscillation in the channel direction and it will pull the beads towards the channel walls instead of keeping the beads in the center of the channel. Therefore many target species can pass by in the center of the channel undetected just like the static case as depicted in Fig. 1(a).

Our solution to get bead oscillation orthogonal to the channel axis was to use a quadrupole magnet. Moser et al. (2009) have shown such a concept making use of removable permanent magnets and two soft magnetic tips connected to an external coil. Our method uses a four coil quadrupole system around a channel. As can be seen in Fig. 2, of the four magnetic poles only two poles are active at the same time. Beads are attracted to the nearest active pole. When the field is switched and the two other poles are activated, the beads will be attracted to one of those poles. When the channel is sufficiently narrow, the nearest active pole for a bead will be on the other side of the channel. By continued switching, flow-orthogonal bead oscillation is then realized.

For a functional bio-separator, this concept has to be parallelized to allow for high volume throughputs. This was done by multiplexing the concept with a square array of soft-magnetic structures covering multiple
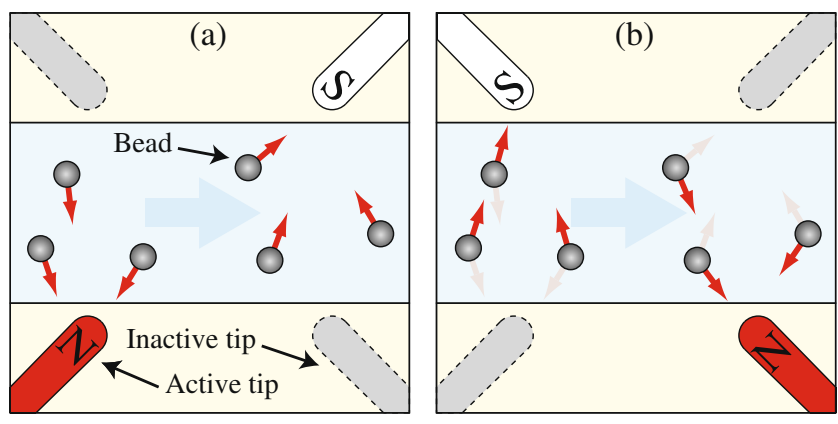

Fig. 2 The oscillating motion is obtained by quadrupolar actuation: beads move towards the active poles that are colored red and white, inactive poles are grey. When comparing the two states (a) and (b), the beads are attracted in the opposite direction with respect to the channel axis 
parallel channels. These magnetic structures function as flux-guides to the externally applied field. The flux density is concentrated in the flux-guides due to their high permeability and mutual alignment. Because the flux-guides consist of elongated objects that form the cross-shapes, they show shape-anisotropic behavior; only the two tips aligned with the externally applied field can effectively magnetize. This gives an array of small local quadrupoles that show the same magnetization as the macro-scale quadrupole with controllable coils.

The quadrupole magnet and the flux-guides were simulated in 2D with the magnetostatics module in Comsol 3.5 as can be seen in Fig. 3. By prescribing the current through the individual coils, the magnetic field could be computed inside the quadrupole airgap where the flux-guide array and channels were positioned. The external quadrupole yoke (not visible in figure) measured $4 \times 4 \mathrm{~cm}$ and the four coils were given an absolute current density of $10^{6} \mathrm{~A} / \mathrm{m}^{2}$, the yoke and flux-guides had a relative permeability of $10^{3}$ and $10^{2}$ respectively. Magnetic insulation was prescribed on
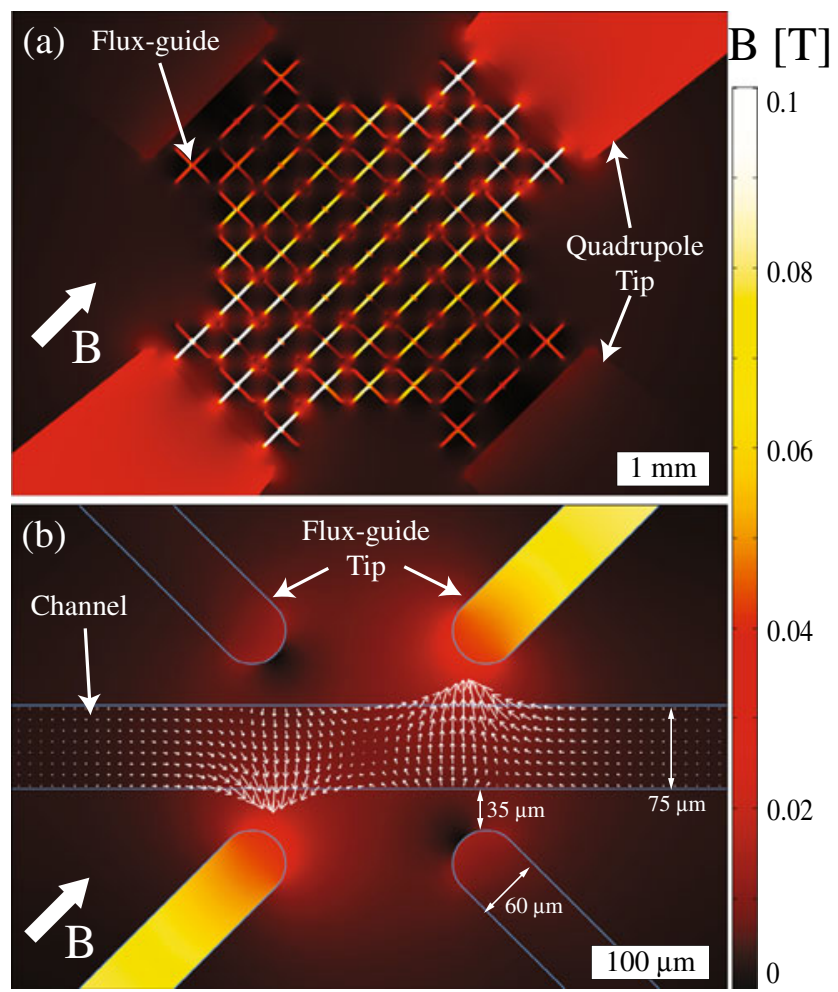

Fig. 3 (a) 2D simulation of the flux density in an array of 61 fluxguides (crosses), the external quadrupole tips can be seen on the four corners, the coils are not visible in the image. The tips of the flux-guides that are aligned to the applied field $B$ show a much higher magnetization which locally creates a high gradient. (b) Zoom-in of the flux-guide tips, arrows show the magnetic force on a bead inside a channel as a function of the position the external boundaries that were placed far away at $\sim 15 \mathrm{~cm}$ to minimize their influence on the solution. The flux-density at the channel positions between the array was around $B \approx 0.1 \mathrm{~T}$ and its gradient $\nabla B^{2} \approx 1 \mathrm{~T}^{2} / \mathrm{m}$. Through Eq. 1 the magnetic force on the bead was known for each location in the channel. By adding the fluid dynamics module, the drag forces and therefore the beads motion could be computed. A steady-state incompressible Navier-Stokes flow was assumed with viscosity $\eta_{\mathrm{f}}=10^{-3} \mathrm{~Pa} \cdot \mathrm{s}$ and density $\rho_{\mathrm{f}}=10^{3} \mathrm{~kg} / \mathrm{m}^{3}$. Walls were modeled with a no-slip boundary condition. Both modules were coupled and parameterized through a Matlab interface. The solution was computed as a function of time using time steps of $10^{-2} \mathrm{~s}$.

The rotation of the field was obtained by a cyclic function for each coil with a mutual phase difference of $90^{\circ}$. For the control signal a function that combined a sine-wave and a constant value was used. For this function a current control ratio $r_{\mathrm{c}}$ was defined as the ratio between time at maximum current and the transition time between two maxima. Figure 4 shows three different cases, it can be seen that for large $r_{\mathrm{c}}$ the signal approached a step-function and for small $r_{\mathrm{c}}$ it approached a sine-wave. This way the field direction could be switched more abruptly or it could rotate more smoothly.

The bead trajectories were taken from the simulations, see Fig. 5 (a movie clip of the simulation can be found in the Supplementary material) and it was found that an increased current ratio results in a narrower trajectory where the bead reaches the other wall faster. This is advantages because it allows for

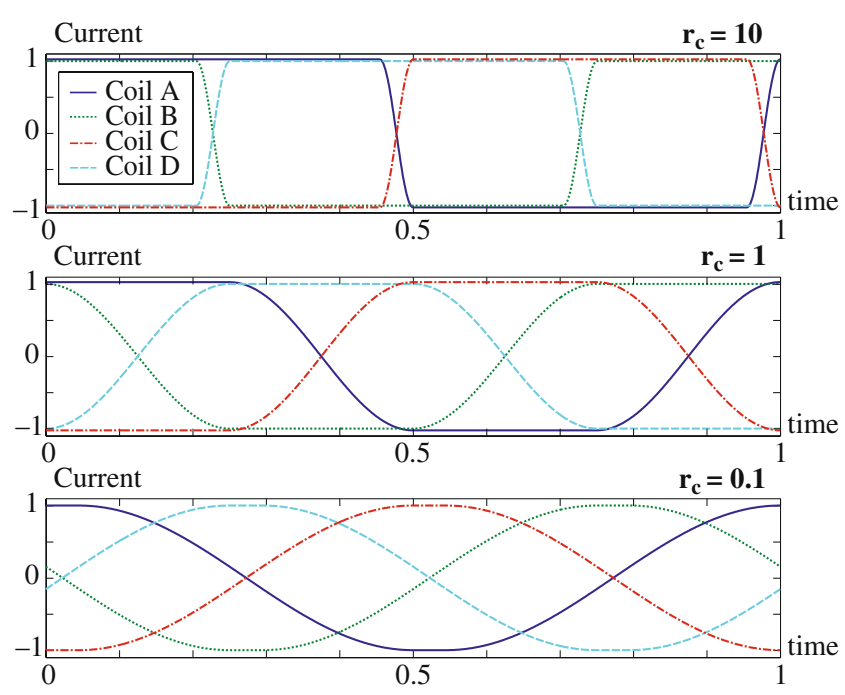

Fig. 4 Normalized control signals for each of the quadrupole coils showing the current as a function of time for different current control ratios 

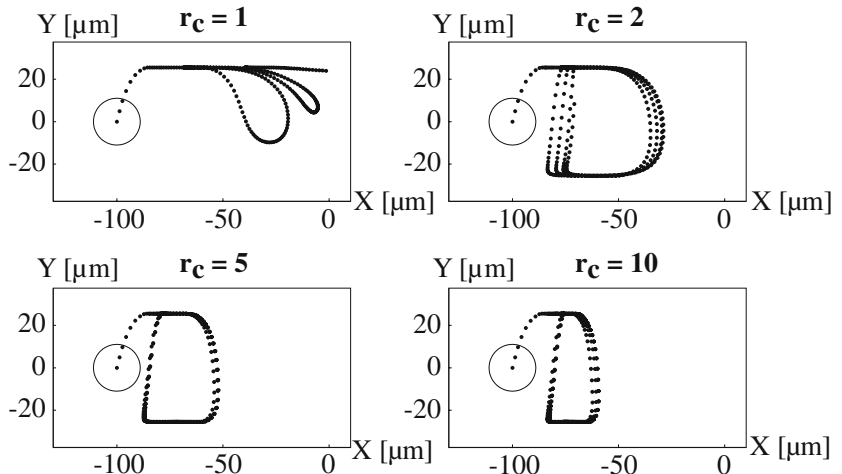

Fig. 5 Resulting bead trajectories for simulations using different control ratios. The bead is drawn at its starting position as a circle. The dots represent the position of the bead at time intervals of $0.01 \mathrm{~s}$. When a bead reaches steady-state, the subsequent cycles overlap

a higher field rotation rate or a higher fluid velocity in the channel and thus in a higher separation rate. The effect is caused by the transition during which the field strength is lower. Therefore the transition time should be minimized. For a bead with a diameter of $22.7 \mu \mathrm{m}$ and $\chi_{\mathrm{b}, \text { eff }}=0.06$ (as was used later in the experiments) velocities up to $500 \mu \mathrm{m} / \mathrm{s}$ were obtained in the simulations.

\section{Fabrication}

For validation of the simulations, a prototype chip was fabricated using polycarbonate (PC) sheets (SABIC LEXAN film). The entire fabrication scheme is shown in Fig. 6. After applying a $25 \mu \mathrm{m}$ thick solid state adhesive (3M laminating adhesive 8141) to the $125 \mu \mathrm{m}$ thick substrate, channels and cavities for the flux-guides were ablated into the substrate and adhesive layer with an excimer laser system (Optec Micromaster, $\mathrm{KrF}$, $248 \mathrm{~nm}$ ). A lid was used to enclose the channels but it also functioned as the electrode in the electroforming process. Therefore $10 \mathrm{~nm}$ of chrome for improved adhesion and then $20 \mathrm{~nm}$ of gold were sputter-coated on a $125 \mu \mathrm{m}$ polycarbonate sheet and then patterned by ablation. Ablation debris was removed together with the release liner from the adhesive after which the lid was bonded to the substrate by hand under a stereomicroscope. Next the nickel flux-guides were grown in the cavities at the channel height by electroforming using a commercial nickel plating kit from Barvic. Finally the tubing was connected to the chip with a 2-component adhesive and a $2 \mathrm{~mm}$ thick polycarbonate interconnect. The completed chip had channels with a cross section of $75 \times 75 \mu \mathrm{m}$ that were placed $0.75 \mathrm{~mm}$ apart. A picture of the completed chip can be found in Fig. 7.

\section{Lid + electrodes}
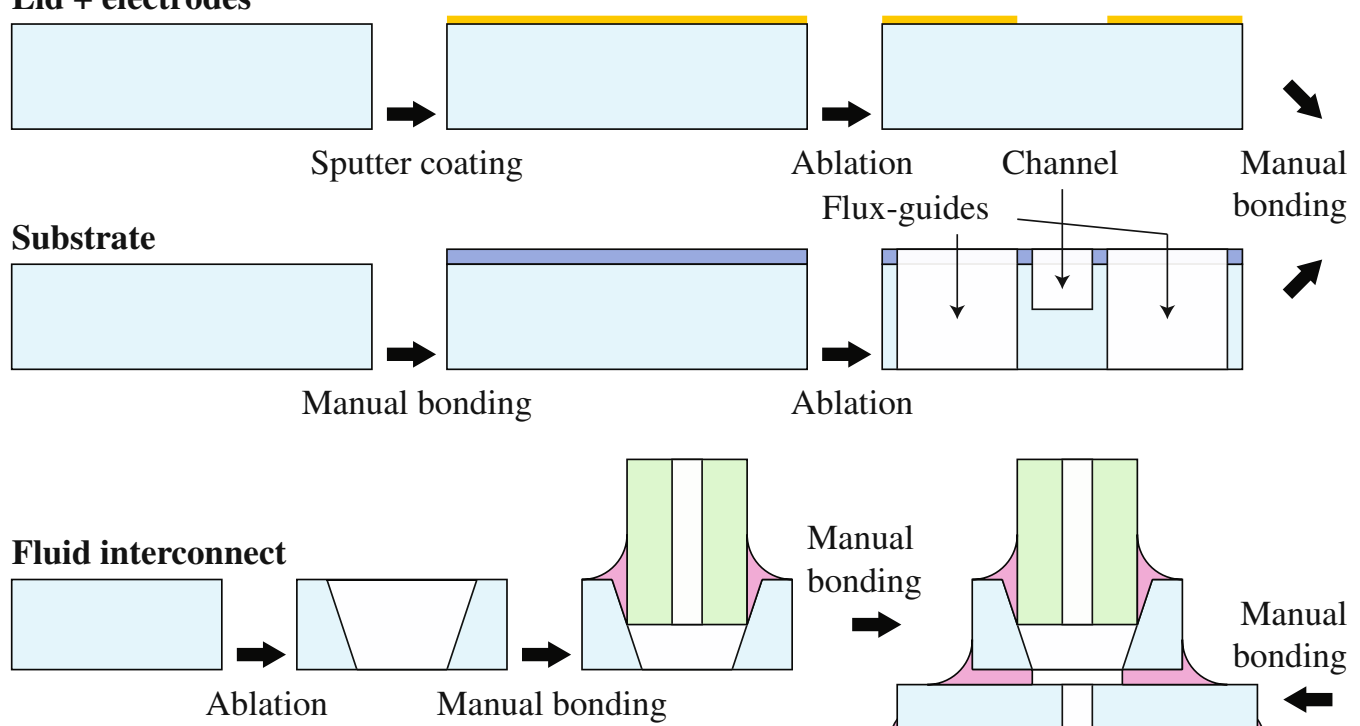

Ablation Manual bonding

\begin{tabular}{|c|c|c|}
\hline PC & Nickel & Solid adhesive \\
\hline Tubing & Gold & 2-Component adhesive \\
\hline
\end{tabular}
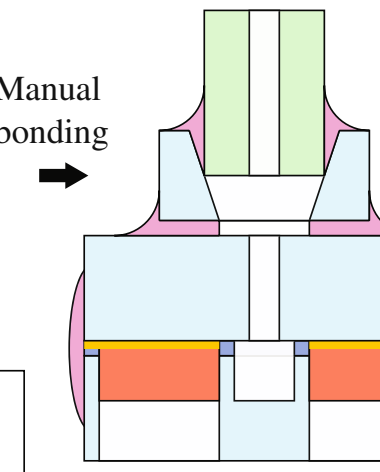

Completed chip

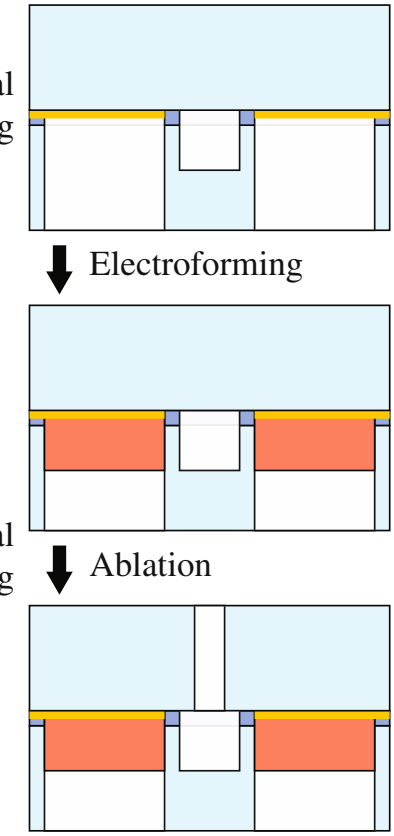

Manual bonding

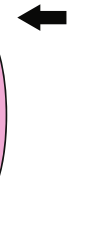

Fig. 6 The subsequent fabrication steps for the prototype chip (not to scale) 

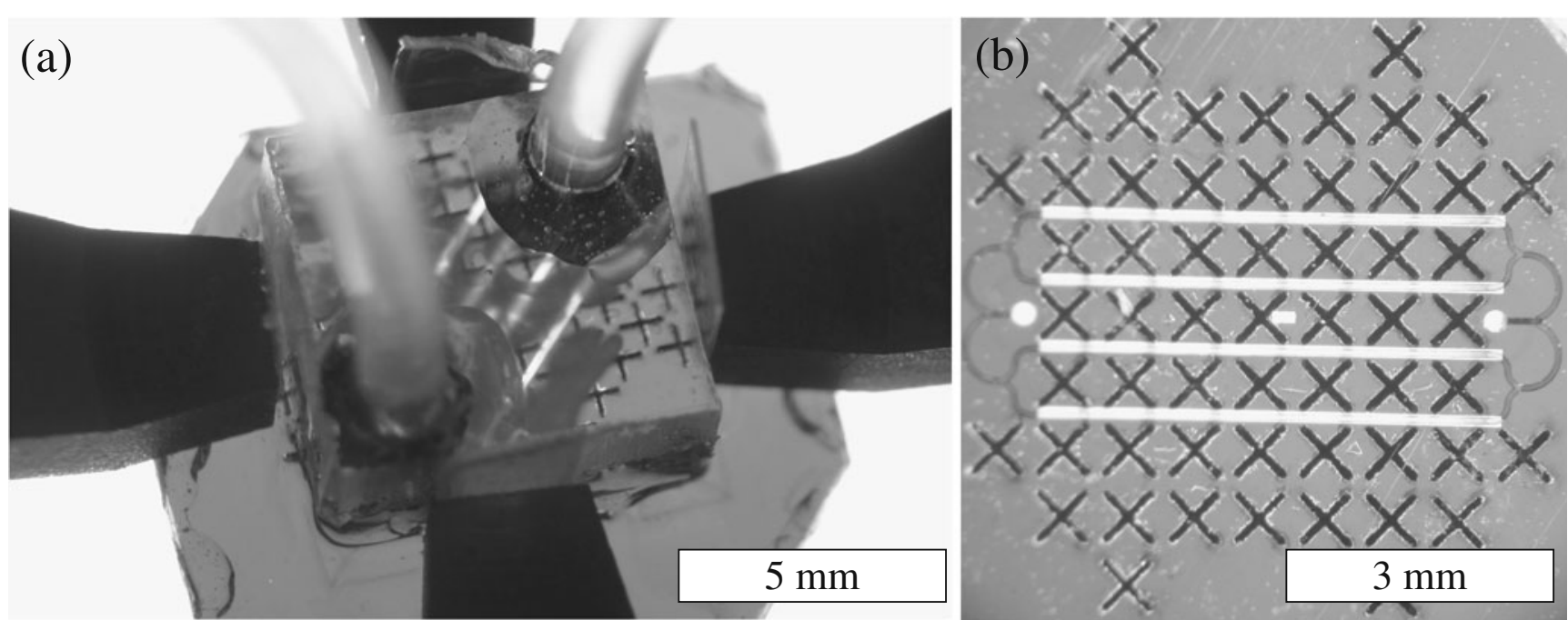

Fig. 7 (a) Photograph of the completed prototype chip in between the four tips of the quadrupole setup. (b) Top view of the flux-guide array and the four parallel channels with their inlet and outlet

\section{Experiments and results}

To confirm the magnetic properties of the electroformed nickel, hysteresis curves of different samples were measured with a vibrating sample magnetometer (VSM). For conformity the samples had the same shape and size as the flux-guides in the prototype chip. Measurements were done on both single flux-guides and on an array of flux-guides. For an optimal flux-guide, the saturation magnetization should be high to allow for a high flux density. The hysteresis should be low so that the direction of magnetization in the flux-guides can easily be changed. The measured values corresponded well with these demands, the saturation magnetization was $430-493 \mathrm{kA} / \mathrm{m}$ and the coercive flux density was 5.5-7.2 $\mathrm{mT}$.

The actual bead oscillation experiments were carried out by placing the prototype chip inside the quadrupole magnet, see Fig. 7. This quadrupole was the same as used in earlier experiments (Petousis et al. 2007) and consisted of a circular soft magnetic yoke with four tips pointing to its center and four coils between each of these tips. The chip was located in the center air gap measuring $7 \times 7 \mathrm{~mm}$.

The field induced by the quadrupole magnet was controlled by prescribing the individual currents through the four coils. This was done with a current controller that was interfaced with a pc with Matlab. The injection of the fluid into the chip was driven by a syringe pump (ProSense NE-1010). The chip was monitored using an optical microscope equipped with a digital camera. Two types of superparamagnetic particles were used: Dynabeads M-280 with a $2.8 \mu \mathrm{m}$ diameter and Spherotech CM-200-10 with a $22.7 \mu \mathrm{m}$ diameter. Figure 8 shows an experiment on a single $22.7 \mu \mathrm{m}$ bead. A real-time movie can be found in the Supplementary material. From the subsequent frames the position of the bead was taken to reconstruct the pathway of the bead as a function of time, see Fig. 9. It was found that the shape of the trajectory depended on the ratio $r_{\mathrm{c}}$. The more smoothly the field was rotated, the wider the trajectory of the bead became, up to two times the channel diameter which compared well with the performed simulations.

To find the maximum frequency at which the bead could still follow, the magnetic field rotation rate was gradually increased. The maximum frequency was $3 \mathrm{~Hz}$

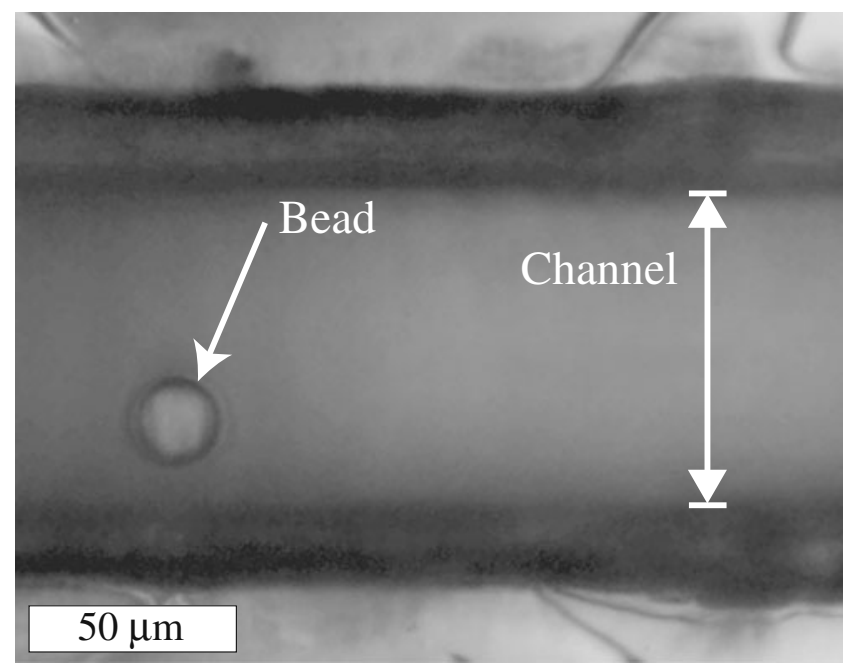

Fig. 8 A $22.7 \mu \mathrm{m}$ bead inside a $75 \mu \mathrm{m}$ wide channel 


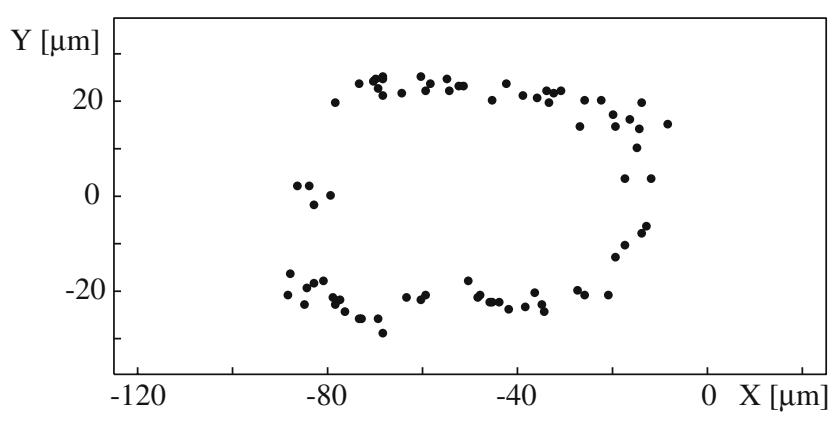

Fig. 9 Measured positions of a bead during 10 cycles measured at $0.2 \mathrm{~s}$ intervals. The field rotated at $0.7 \mathrm{~Hz}$ with a current ratio of $r_{\mathrm{c}}=2$

at $r_{\mathrm{c}}=2$ which gave an average velocity of $450 \mu \mathrm{m} / \mathrm{s}$. At higher frequencies, the bead would still oscillate at the prescribed frequency, but it would not reach both walls anymore. When comparing this to the simulations, it was found that in the experiment, the maximum frequency was a factor 3 higher. One reason was that the simulations were performed in 2D where the bead could not be modeled as a sphere. Instead the crosssection of the bead was taken. This resulted in the drag force being slightly higher than in the experiments.

Experiments on multiple $1 \mu \mathrm{m}$ beads resulted in the formation of chains of approximately $25 \mu \mathrm{m}$ long. These chains moved in trajectories very similar to the single bead case. The formation of chains will decrease the drag force as the frontal surface area per bead is decreased, this allows for higher bead velocities. These chains however, also rotated along with the prescribed field, similar as reported earlier (Petousis et al. 2007). This rotation might enhance mixing of the fluid which could improve the capture efficiency in the case of a bio-separation.

\section{Conclusion}

We have shown a new concept for the oscillation of superparamagnetic beads orthogonal to the axis of a microfluidic channel. This concept can easily be multiplexed for high throughput applications while electric or magnetic interconnects can be avoided. Our polycarbonate based fabrication combines both the fluidic and magnetic functionality within a single layer to maximize magnetic efficiency. A working prototype chip was successfully manufactured and experiments corresponded well to simulations. Furthermore, control of the bead trajectory shape was possible. This allows for application as a bio-separator or fluidic mixer.
Future work will consist of in-flow experiments. Already some preliminary simulations inside a flow where performed which resulted in stable bead oscillation with fluid velocities up to $0.2 \mathrm{~mm} / \mathrm{s}$ (an example can be found in the Supplementary material). After this has been validated experimentally, the concept can be tested using an immunoassay. A second possible functionality might be to use the oscillating beads as active mixer elements. Additionally, the manipulation concept should be characterized in more detail using different types of beads and geometries for the channels and flux-guides.

Open Access This article is distributed under the terms of the Creative Commons Attribution Noncommercial License which permits any noncommercial use, distribution, and reproduction in any medium, provided the original author(s) and source are credited.

\section{References}

T. Aytur, J. Foley, M. Anwar, B. Boser, E. Harris, P.R. Beatty, A novel magnetic bead bioassay platform using a microchipbased sensor for infectious disease diagnosis, J. Immunol. Methods 314(1-2), 21-29 (2006)

R. Derks, A. Dietzel, R. Wimberger-Friedl, M. Prins, Magnetic bead manipulation in a sub-microliter fluid volume applicable for biosensing, Microfluidics and Nanofluidics 3(2), 141149 (2007)

J. Do, C.H. Ahn, A polymer lab-on-a-chip for magnetic immunoassay with on-chip sampling and detection capabilities, Lab on a Chip 8(4), 542-549 (2008)

G. Fonnum, C. Johansson, A. Molteberg, S. Mø rup, E. Aksnes, Characterisation of Dynabeads ${ }^{\circledR}$ by magnetization measurements and Mössbauer spectroscopy, J. Magn. Magn. Mater. 293(1), 41-47 (2005)

A.G. Gehring, P.L. Irwin, S.A. Reed, S.-I Tu, P.E. Andreotti, H. Akhavan-Tafti, R.S. Handley, Enzyme-linked immunomagnetic chemiluminescent detection of escherichia coli O157:H7, J. Immunol. Methods 293(1-2), 97-106 (2004)

M.A.M. Gijs, Magnetic bead handling on-chip: new opportunities for analytical applications, Microfluidics and Nanofluidics 1(1), 22-40 (2004)

S.G. Gundersen, I. Haagensen, T.O. Jonassen, K.J. Figenschau, N. de Jonge, A.M. Deelder, Magnetic bead antigen capture enzyme-linked immunoassay in microtitre trays for rapid detection of schistosomal circulating anodic antigen, J. Immunol. Methods 148(1-2), 1-8 (1992)

K. Gunnarsson, P.E. Roy, S. Felton, J. Pihl, P. Svedlindh, S. Berner, H. Lidbaum, S. Oscarsson, Programmable motion and separation of single magnetic particles on patterned magnetic surfaces, Adv. Mater. 17(14), 1730-1734 (2005)

G.P. Hatch, R.E. Stelter, Magnetic design considerations for devices and particles used for biological high-gradient magnetic separation (hgms) systems, J. Magn. Magn. Mater. 225(1-2), 262-276 (2001)

T. Lund-Olesen, B.B. Buus, J.G. Howalt, M.F. Hansen, Magnetic bead micromixer: influence of magnetic element geometry and field amplitude, J. Appl. Phys. 103(7), 07E902-3 (2008) 
A. Manz, N. Graber, H.M. Widmer, Miniaturized total chemical analysis systems: a novel concept for chemical sensing, Sensor. Actuat. B-Chem. 1(1-6), 244-248 (1990)

Y. Moser, T. Lehnert, M.A.M. Gijs, Quadrupolar magnetic actuation of superparamagnetic particles for enhanced microfluidic perfusion, Appl. Phys. Lett. 94(2), 022505 (2009)

I. Petousis, E. Homburg, R. Derks, A. Dietzel, Transient behavior of magnetic micro-bead chains rotating in a fluid by external fields, Lab on a Chip 7, 1746-1751 (2007)
Q. Ramadan, V. Samper, D. Poenar, C. Yu, Magnetic-based microfluidic platform for biomolecular separation, Biomedical Microdevices 8(2), 151-158 (2006)

A. Rida, M.A.M. Gijs, Manipulation of self-assembled structures of magnetic beads for microfluidic mixing and assaying, Anal. Chem. 76(21), 6239-6246 (2004)

K. Smistrup, B.G. Kjeldsen, J.L. Reimers, M. Dufva, J. Petersen, M.F. Hansen, On-chip magnetic bead microarray using hydrodynamic focusing in a passive magnetic separator, Lab on a Chip 5(11), 1315-1319 (2005) 